\title{
Broad-Band Parametric Deamplification of Quantum Noise in an Optical Fiber
}

\author{
R. M. Shelby, M. D. Levenson, S. H. Perlmutter, R. G. DeVoe, and D. F. Walls ${ }^{(a)}$ \\ IBM Almaden Research Center, San Jose, California 95120 \\ (Received 27 May 1986)
}

\begin{abstract}
Broad-band squeezed states of light are generated by forward nondegenerate four-wave mixing in a single-mode optical fiber cooled below $4.2 \mathrm{~K}$. The minimum total noise level was $(12.5 \pm 0.5) \%$ below the standard quantum limit.
\end{abstract}

PACS numbers: $42.50 . \mathrm{Dv}, 06.30 . \mathrm{Lz}, 42.65 . \mathrm{Ky}$

Modern theories of optical noise indicate that certain radiation states may produce smaller fluctuations of photoelectric detector current than the familiar coherent states. ${ }^{1-3}$ Various nonlinear optical processes have been proposed for generating such "squeezed states" of light by parametric deamplification of vacuum fluctuations, but only one-backwards four-wave mixing in an atomic beam contained in an optical cavity-has previously proved successful. ${ }^{4}$ We have now demonstrated the parametric deamplification of electromagnetic vacuum fluctuations in a travelingwave geometry by use of a single-mode optical fiber. Although the squeezing reported here could only be detected between 40 and $60 \mathrm{MHz}$ because of lightscattering noise, in principle the bandwidth of such traveling-wave squeezing devices extends from 0 to $100 \mathrm{GHz}$, limited only by phase-matching considerations.

Squeezed-state generation in single-mode optical fibers has been discussed previously. ${ }^{5-7}$ Two acoustooptic phenomena-stimulated Brillouin oscillation and spontaneous guided acoustic-wave Brillouin scattering - must be overcome for such a technique to be effective $^{5,8}$ of these the latter is more serious; we have been forced to immerse the entire 114-m optical fiber in superfluid helium to suppress the spontaneous Brillouin scattering. Even so, the noise due to light scattering is only reduced, not eliminated, and constitutes the main phenomenon that increases the detected fluctuations. Cooling the fiber, however, lowers the threshold for stimulated Brillouin oscillation to roughly $10 \mathrm{~mW}$. The $\sim 200-\mathrm{mW}$ pump beam can be propagated through the fiber only because its power has been divided among $\sim 25$ frequency components with a phase modulator. ${ }^{9,10}$ The full theory of squeezed-state generation by four-wave mixing with such a multifrequency pump will be presented separately, but when the correct detection strategy is employed, the noise suppression is the same as the single-frequency case in Ref. 6.

The photodetector noise current at frequency $\delta$ is generated by heterodyne beats of the pump frequency $\omega^{P}$ with the upper and lower noise sidebands at $\omega^{P}+\delta$ and $\omega^{P}-\delta$ which are arbitrarily designated as the signal $\left(\omega^{S}=\omega^{P}+\delta\right)$ and idler $\left(\omega^{I}=\omega^{P}-\delta\right)$ frequencies.
Four-wave mixing in the fiber couples $\omega^{S}$ and $\omega^{I}$ together, creating broad-band squeezed states. The pump frequency need not be constant; in our case it oscillated sinusoidally. At the fiber output, the phaseshifted local oscillator is derived from the frequencymodulated pump. When the resulting beam is incident on a detector, the fluctuations in the photocurrent at frequency $\delta$ are not affected by the phase modulation, and the same squeezing is obtained as in the singlefrequency case. ${ }^{6}$

The actual scheme of the experiment appears in Fig. 1. A stabilized single-frequency $647-\mathrm{nm}$ krypton ion laser beam is phase modulated in a resonant doublepass $\mathrm{LiTaO}_{3}$ modulator. The resulting pump beam has constant total power, but many frequency components:

$$
\begin{aligned}
E_{p}(t) & =\frac{1}{2} E_{0} \exp \left[i\left(\omega_{0} t+M \sin \omega_{M} t\right)\right]+\text { c.c. } \\
& =\frac{1}{2} E_{0} e^{i \omega_{0} t} \sum_{n=-\infty}^{\infty} J_{n}(M) e^{i n \omega_{M} t}+\text { c.c. }
\end{aligned}
$$

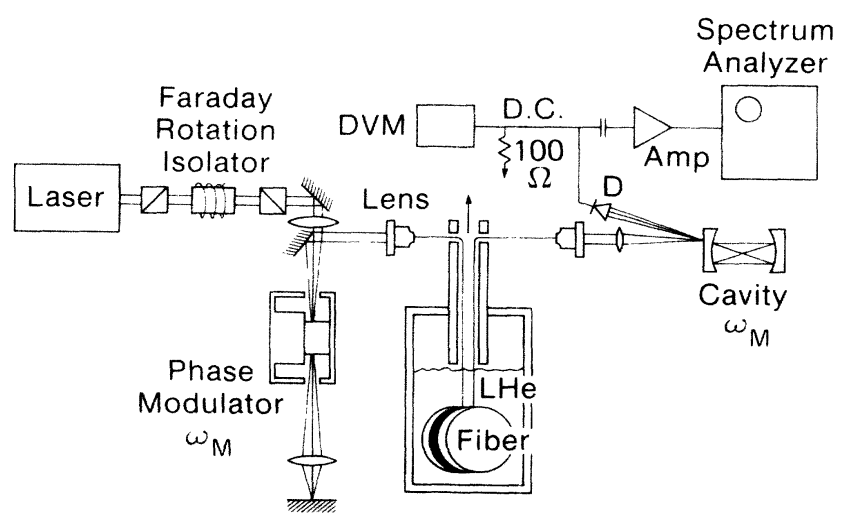

FIG. 1. Schematic of the experimental apparatus. The two-pass resonant phase modulator is driven at exactly the mode-spacing frequency of the phase-shifting cavity. The coil of $5-\mu \mathrm{m}$-core single-mode fiber is immersed in superfluid helium with the input and output lengths cooled by helium gas. The beam from the fiber is reflected by the phase-shifting cavity and detected by the EG\&G model FND-100 photodiode labeled D. The dc current from the diode is monitored by a digital voltmeter labeled DVM, while the ac noise signals are displayed on an rf spectrum analyzer. 
where $\omega_{M}=748.44 \mathrm{MHz}$ is the modulation frequency, $M \approx 10$ is the modulation index, and $J_{n}$ is the $n$ thorder Bessel function of the first kind. ${ }^{11}$ The pump light is then coupled (along with vacuum fluctuations) into $114 \mathrm{~m}$ of bare single-mode optical fiber in a helium cryostat. If we neglect the loss and group-velocity dispersion of the fiber, it can be shown that such a pump field propagates through the fiber unmodified except for an overall nonlinear phase shift. ${ }^{6}$ The noise current produced by a photodiode is due to beats between noise-sideband amplitudes and each pumpfrequency component. In the fiber, the noisesideband amplitudes are coupled together by the optical nonlinearity. Consider signal and idler amplitudes at frequencies $\omega_{n}^{S}=\omega_{0}+n \omega_{M}+\delta$ and $\omega_{n}^{I}=\omega_{0}$ $+n \omega_{M}-\delta$, respectively, where $\delta$ is the (rf) noise frequency. The noise-sideband amplitude operators, $\hat{E}_{n}^{S}$ and $\hat{E}_{n}^{I}$, are coupled together according to

$$
\begin{aligned}
& \frac{\partial \hat{E}_{j}^{I}}{\partial z}=\frac{i r}{l}\left[2 \hat{E}_{j}^{I}+e^{i 2 r / l} \sum_{n=-\infty}^{\infty} J_{j+n}(2 M) \hat{E}_{n}^{S^{\dagger}}\right], \quad \text { (2a) } \\
& \frac{\partial \hat{E}_{j}^{S^{\dagger}}}{\partial z}=\frac{-i r}{l}\left[2 \hat{E}_{j}^{S^{\dagger}}+e^{-i 2 r / l} \sum_{n=-\infty}^{\infty} J_{j+n}(2 M) \hat{E}_{n}^{I}\right],
\end{aligned}
$$

where $r=K\left|E_{0}\right|^{2} l, \quad l$ is the fiber length, and $K$ $=(12 \pi \omega / n c) \chi^{(3)}$ is the nonlinear coupling. ${ }^{6}$

The output beam from the fiber is then reflected from an optical cavity with axial mode spacing equal to the modulation frequency. This cavity converts the pump to a local oscillator by equally attenuating each frequency component and phase shifting each frequency component by $\theta .^{6,7}$ The finesse of this cavity is approximately 35 and its reflectivity at zero offset is $6 \%$. The signal and idler sidebands are only slightly attenuated and not phase shifted. The resulting beam contains strong local-oscillator waves at the frequencies $\omega^{0}+n \omega_{M}$ and a continuum of vacuum fluctuations, potentially squeezed by the four-wave mixing interaction. This beam then falls on an FND-100 photodetector in a resonant circuit tuned to approximately $55 \mathrm{MHz}$. The Johnson noise in this circuit is typically $16 \mathrm{~dB}$ less than the optical noise. The dc current produced by the photodiode (roughly $8 \mathrm{~mA}$ ) generates a voltage on a $100-\Omega$ resistor which is then measured by a digital voltmeter. The ac components are filtered to remove any components near or above $\omega_{M}$, amplified, and displayed on a spectrum analyzer. The fluctuating noise current is due to the interference of the local oscillators and noise sidebands. The Fourier component of the optical power that produces the fluctuating current at frequency $\delta$ is

$$
\begin{aligned}
\hat{I}(\delta) & =\left|E_{\mathrm{LO}}\right| \sum_{k=-\infty}^{\infty} J_{k}(M)\left[e^{-i(\theta+r)} \hat{E}_{k}^{I}(l)+e^{i(\theta+r)} \hat{E}_{k}^{S^{\dagger}}(l)\right]+\text { H.c. } \\
& =\left|E_{\mathrm{LO}}\right| \sum_{k=\infty}^{\infty} J_{k}(M)\left\{\left[\hat{E}_{k}^{I}(0)+\hat{E}_{k}^{S^{\dagger}}(0)\right](\cos \theta+2 r \sin \theta)-i\left[\hat{E}_{k}^{I}(0)-\hat{E}_{k}^{S^{\dagger}}(0)\right] \sin \theta\right\}+\text { H.c. }
\end{aligned}
$$

where $\hat{E}_{n}^{I}(0)$ and $\hat{E}_{n}^{S}(0)$ are the operators at the input to the fiber which characterize the vacuum fluctuations at the frequencies $\omega_{n}^{I}$ and $\omega_{n}^{S}$, respectively, and $\left|E_{\mathrm{LO}}\right|^{2}$ is the total local-oscillator intensity. The electrical noise power produced by the detector is proportional to $\left\langle|\hat{I}(\delta)|^{2}\right\rangle$ which can be calculated from this expression. ${ }^{6}$ Almost all of the cross terms cancel because of various Bessel-function identities, yielding a functional form identical to that in Ref. 6 .

A servo system held the resonances of the phaseshifting cavity at a variable offset from the frequency components of the pump. The servo-stabilized cavity maintained a phase shift $\theta$ constant within $3^{\circ}$ for many minutes of operation. Other parameters-such as the transmitted pump power-were similarly constant enough for reproducible data collection. It proved important to remove the protective jacket from the fiber to avoid light scattering or loss at low temperatures, and to cool the input and output fibers with flowing helium gas. The ratio of the detector current to the transmitted power indicated an overall detection quantum efficiency of $(80 \pm 5) \%$; however, modulation experiments indicate that the local-oscillator-sideband spatial overlap factor is only $63 \%$. Thus, only $\sim 50 \%$ of the noise suppression can actually be detected.

In a typical experiment, the laser power was set to produce a transmitted pump wave of $100-200 \mathrm{~mW}$. With the phase shift set equal to zero, the modulation frequency was adjusted to minimize the noise level measured at the detection frequency, typically 55 $\mathrm{MHz}$. This procedure insures that the modulation frequency exactly equals the cavity mode spacing, as otherwise the phase noise of the pump laser would appear as amplitude noise on the detector. ${ }^{12}$ The phase shift was then varied and the noise level and dc current for each setting were logged by computer. Ten to twenty measurements were made of the detector current at each phase, and 1000 noise measurements made during a 3-sec dwell time. The spectrum-analyzer resolution bandwidth was $300 \mathrm{kHz}$, and the video bandwidth 30-3000 Hz. A computer sampled and displayed the noise and current levels along with their ratio.

The current at the minimum noise-to-current ratio was noted, and the noise level of the standard quantum limit immediately determined by focusing of suf- 
ficient filtered incandescent light onto the diode to yield that current. ${ }^{13}$ It proved important to remove all of the long-wavelength radiation from the incandescent beam. Readings were also taken bracketing the current levels seen in the experiment. In a subsidiary experiment, the noise level of the unmodulated laser was also compared to the incandescent source over a range of detector currents. The unmodulated laser gave a noise level systematically $(2.0 \pm 1.0) \%$ higher than the incandescent source. We have taken the incandescent-source noise level as our definition of the standard quantum limit.

Typical ratios of the noise and current are displayed as functions of local-oscillator phase in Fig. 2. The error bars reflect the standard deviations of the distribution of measurements. The radius of the dots reflect three times the standard deviations of the means computed according to the Student $t$ distribution. ${ }^{14}$ This latter quantity correctly estimates the statistical uncertainty of the average of these measurements. The solid line is our experimental realization of the standard quantum limit. Its width reflects the $0.2 \%$ estimated uncertainty of the noise level for a coherent state. At the minimum of the ratio, the noise level of the light produced by our optical-fiber system is ( 12.5 $\pm 0.5) \%$ below the measured coherent-state level. This 25-standard-deviation shift verifies that quantum fluctuations can indeed be deamplified by forward parametric mixing in an optical fiber with $>99.9 \%$ confidence.

The curved line is a plot of the function

$$
4 V=1+\eta \frac{4 r^{2}}{L^{2}}\left(1-e^{-L}-L e^{-L}\right)(1-\cos 2 \theta)+\frac{2 r}{L}\left(1-e^{-L}\right) \sin 2 \theta+g(\delta) r(1-\cos 2 \theta)+\operatorname{ar}(1+\cos 2 \theta),
$$

which is the predicted ratio of the noise power in our experiment to that of a coherent state of equal flux. In Eq. (4), $L=0.2$ is the attenuation of the fiber, $r$ is the squeeze parameter as in Ref. $6, \theta$ is the phase shift, and $\eta=0.5$ is the quantum efficiency. The last term on the right parametrizes a small excess amplitude noise. The quantity $g(\delta)=1.16$ (at $56.25 \mathrm{MHz}$ ) represents phase noise produced by low-frequency light scattering in the fiber. ${ }^{7,8,15}$ While low temperatures reduce $g$, the minimum value we observe is not as much less than the room-temperature value $(g=8)$ as one might expect if this noise were due to thermally excited mechanical vibrations. In this fiber, we had employed the classical noise-squeezing technique to show that $r \sim 0.03 / P$ where $P$ is in watts and $l$ in meters. With the correct value of the excess noise $(a=0.16)$ and squeeze parameter $(r=0.6)$, Eq. (4) fits the points of Fig. 2 excellently. Similar results were obtained at different detection frequencies and various pump powers.

Figure 3 shows the spectrum of photocurrent noise produced by a broad-band detector when the phase $\theta$ is

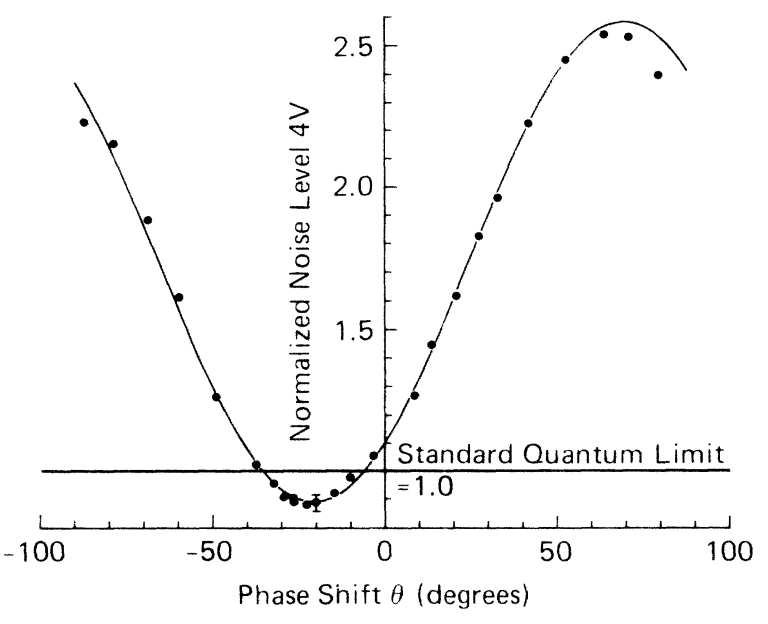

FIG. 2. The ratio of the rf noise at $56.25 \mathrm{MHz}$ to the dc current as a function of phase shift $\theta$. The ratios have been normalized to those for an incandescent source producing the same current. The standard quantum limit determined in this manner is unity on this graph. The width of the line reflects the $0.2 \%$ experimental uncertainty in this measurement. The solid circles reflect the noise levels obtained from the experiment in Fig. 1 with $220 \mathrm{~mW}$ of pump radiation. The radius of the circles is three times the $0.4 \%$ standard deviation of the means of the measurements while the error bracket is the $3 \%$ standard deviation of the distribution of all the measurements at phase $\theta$. The curve is a fit by Eq. (4) with $r=0.62, g=1.16, \eta=0.5, L=0.2$, and $a=0.16$. The spectrum-analyzer resolution bandwidth was $300 \mathrm{kHz}$ and video bandwidth was $30 \mathrm{~Hz}$. 
at the minimum of Fig. 2. The noise level has been normalized to that of an incandescent source producing exactly the same photocurrent. The electrical noise in the broad-band photodetector is $19 \%$ of the total noise measured with the incandescent source. Between 41 and $49 \mathrm{MHz}$ and between 51 and $59 \mathrm{MHz}$, the noise level is clearly below that of the experimentally realized standard quantum limit. The frequency dependence of this noise reflects the spectrum of spontaneous light scattering parametrized by $g(\delta)$. Peaks at 37,50 , and $60 \mathrm{MHz}$ are caused by known guided acoustic-wave Brillouin scattering modes of the fiber, but the excess noise between the peaks is probably related to two-level tunneling modes in the glass. ${ }^{16}$ Figure 3 clearly shows that noise below the standard quantum limit exists over an appreciable bandwidth.

The excellent precision of this experiment permits some of the predicted properties of squeezed states to be verified. We have, for example, inserted a variable neutral density filter between the cavity and the detector and shown that the decrease in noise level below the standard quantum limit varies linearly with filter transmission. The squeezed-state experiments to date have not realized large noise suppressions, but neither have they documented any fundamental limitations to the squeezing phenomenon. Rather, each has shown that the degree of quantum noise suppression is limited by some material-dependent light scattering process that adds excess noise at the detected frequency. Systems without such processes should reward those who search for them with much improved noise suppression.

The authors acknowledge highly useful interactions with Dr. Margaret Reid, Dr. Joseph Weisocki, Dr. Richard G. Brewer, and Mr. Don Horne. This research was partly supported by the Office of Naval Research.

(a) Permanent address: University of Waikato, Hamilton, New Zealand.

${ }^{1}$ D. F. Walls, Nature (London) 306, 141 (1983).

${ }^{2}$ C. M. Caves, Phys. Rev. D 23, 1693 (1981).

${ }^{3}$ H. P. Yuen, Phys. Rev. A 13, 2226 (1976).

${ }^{4}$ R. E. Slusher, L. W. Hollberg, B. Yurke, J. C. Mertz, and J. F. Valley, Phys. Rev. Lett. 55, 2409 (1985).

${ }^{5}$ M. D. Levenson, R. M. Shelby, M. D. Reid, D. F. Walls, and A. Aspect, Phys. Rev. A 32, 1550 (1985).

${ }^{6}$ M. D. Levenson, R. M. Shelby, and S. H. Perlmutter, Opt. Lett. 10, 514 (1985).

${ }^{7}$ R. M. Shelby, M. D. Levenson, D. F. Walls, A. Aspect, and G. J. Milburn, Phys. Rev. A 33, 4008 (1986).

${ }^{8}$ R. M. Shelby, M. D. Levenson, and P. W. Bayer, Phys. Rev. Lett. 54, 939 (1985).

${ }^{9}$ R. G. DeVoe, J. Hofnagel, and R. G. Brewer, to be published.

${ }^{10}$ D. Cotter, J. Opt. Commun. 4, 10 (1983).

${ }^{11} \mathrm{P}$. Panter, Modulation, Noise, and Spectral Analysis (McGraw-Hill, New York, 1965), Chap. 7.

12R. G. DeVoe and R. G. Brewer, Phys. Rev. A 30, 2827 (1984).

${ }^{13}$ R. M. Gagliaro and S. Karp, Optical Communications (Wiley, New York, 1971), Chap. 5.

${ }^{14}$ R. Langley, Practical Statistics (Dover, New York, 1971), p. 160.

${ }^{15}$ D. F. Walls, J. Phys. A 6, 496 (1973).

${ }^{16}$ P. W. Anderson, B. I. Halperin, and C. M. Varma, Philos. Mag. 25, 1 (1972). 